\title{
Multi-carrier Equalization by Restoration of RedundancY (MERRY) for Adaptive Channel Shortening in Multi-carrier Systems
}

\author{
Samir Abd Elghafar ${ }^{1,2}$, Salaheldin M. Diab ${ }^{1}$, Bassiouny M. Sallam ${ }^{1}$, Moawad I. Dessouky ${ }^{1}$, \\ El-Sayed M. El-Rabaie ${ }^{1}$, Fathi E. Abd El-Samie ${ }^{1}$ \\ 1Department of Electronics and Electrical Communications, Faculty of Electronic \\ Engineering, Menoufia University, Menouf, 32952, Egypt. \\ 2Faculty of Engineering, \\ Department of Electrical Engineering,Jazan University,Jazan, Saudi Arabia
}

\begin{abstract}
This paper proposes a new blind adaptive channel shortening approach for multi-carrier systems. The performance of the discrete Fourier transform-DMT (DFT-DMT) system is investigated with the proposed DST-DMT system over the standard carrier serving area (CSA) loop1. Enhanced bit rates demonstrated and less complexity also involved by the simulation of the DST-DMT system.
\end{abstract}

\section{Keywords}

DFT, DMT, DST.

\section{INTRODUCTION}

Multi carrier modulation, particularly Discrete Multi-tone (DMT) modulation, is one of the most prominent modulation methods for high-speed digital communications. For the channel in the DMT system separated into self- regulating sub-channels. The full amount of bits transmitted over the channel would be the total amount of the bits transmitted in each sub-channel. The binary input data are passed onto a set of parallel, independent sub-channels, each of which is assigned a fixed number of bits during startup or system initialization. Given the measured SNR of each sub-channel during startup, the number of bits for each sub-channel is then determined [1]. Each sub-channel is encoded into a quadrature amplitude modulation (QAM) constellation of the appropriate size. For example, if four bits are assigned to tone $i$, then tone $i$ will use a 16QAM constellation for encoding. The modulation function, IFFT, converts the encoded binary data in QAM constellation format from frequency domain to time domain for transmission. The DMT demodulation regress the time domain signal back to frequency domain data.

A number of $v$ samples is inserted as a guard period at the start of each DMT symbol of to decrease inter-symbol interference (ISI) at $v \geq \mathrm{L}-1$, where $\mathrm{L}$ is the length of the channel. Intercarrier interference (ICI) can be reduced when the guard period is a cyclic prefix [2]. The guard period reduces the channel throughput by a factor of $\mathrm{N} /(\mathrm{N}+v)$, where $\mathrm{N}$ is both the symbol length and FFT length. This factor decreases as $v$ turns into large comparative to $\mathrm{N}$, in order that the presentation failure care for unreasonable. Hence, $v$ is chosen to be relatively small compared to $\mathrm{N}$. In the field, however, ADSL and VDSL channel impulse responses can exceed N/16 samples [3].

DOI : 10.5121/ijnsa.2013.5606 
Time-domain equalizer (TEQ) is used for shortening. Accordingly, the DMT equalizer construction consists of a flow of a single TEQ to shorten the L, a fast Fourier transform (FFT) to present multi carrier demodulation, and a single-tap frequency-domain equalizer (FEQ) per tone to correct frequency distortion in the shortened channel. Different DMT TEQ design methods optimize FIR coefficients based on training data under different criteria. The method of Minimum Mean Squared Error (MMSE) design is one of the beneficial methods [4,5,6,7] to minimize the mean square error among the output of the route consisting of the channel and TEQ filter and the output of a controlled rout consisting of a transmission delay $\Delta$ and a purpose filter. The method of maximum shortening SNR (MSSNR) [3] deals with a challenge to reduce inter-symbol interference (ISI) in the time domain. The MSSNR method aims at increasing the quotient of the energy of the used channel impulse response within an object window of $v+1$ samples to that outer the object window. The Minimum-ISI (Min-ISI) method works by weighting the ISI in the frequency domain [4], for example, to set the ISI in vacant and noisy sub-channels. The traditional joint method of TEQ-FEQ structure equalizes all sub-channels that possibly bound bit rate presentation. An alternate receiver architecture has been proposed in [8], in which to relocate the TEQ procedures to the FEQ. The joint TEQ-FEQ would provide a multi-tap FEQ where each subchannel is discretely equalized. Another alternative structure has been proposed in [9], in which the authors proposed the FEQ transfer to the TEQ to give up complex-valued time-domain equalizer filter banks. Joint equalization methods give up higher data rates for the downstream ADSL task [10].

Multi-carrier modulation (MCM) techniques including discrete multi-tone (DMT) and orthogonal frequency division multiplexing (OFDM) have been used extensively in many wireless network standards, such as IEEE 802.1 la, IEEE 802.16a, and wire-line digital communication systems, such as (ADSL) $[11,12,13]$. MCMs based on discrete Fourier transform (DFT). They utilize the complex exponential functions group such as orthogonal source. Particularly, in discrete multitone (DMT) systems, modulation by the IFFT and demodulation by the FFT create orthogonal sub-channels [14]. But, the exponential functions are not the rare orthogonal source so as to construct baseband multicarrier signals. A single trigonometric function can be used to perform as an orthogonal source to apply to the MCM system, and this system can be synthesized using a discrete sine transform (DST) [12].

TEQ design methods have more complications, and need a channel approximation. The proposed algorithm in [15] which is a blind adaptive channel shortening algorithm solves the complexity problem. This algorithm has low complexity and is globally convergent with only a small loss in bit rate [15]. So in this paper, we can use the DST; along with its inverse as a replacement for the inverse fast Fourier transform / fast Fourier transform (IFFT/FFT) stages in DMT systems in order to enhance the performance of the algorithm in [15] and achieve a higher bit rate with low complexity.

\section{Scheme Design}

The scheme design is shown in Figure 1. Every one of the $N$ frequency bins is modulated by a QAM signal, while frequently a number of bins are left as unacceptable carriers [4]. Modulation is performed via an IFFT, and demodulation is accomplished via FFT. The TEQ is used to decrease the channel length, and the follow-on reduced efficient channel is matched by a frequency-domain equalizer (FEQ). 
International Journal of Network Security \& Its Applications (IJNSA), Vol.5, No.6, November 2013

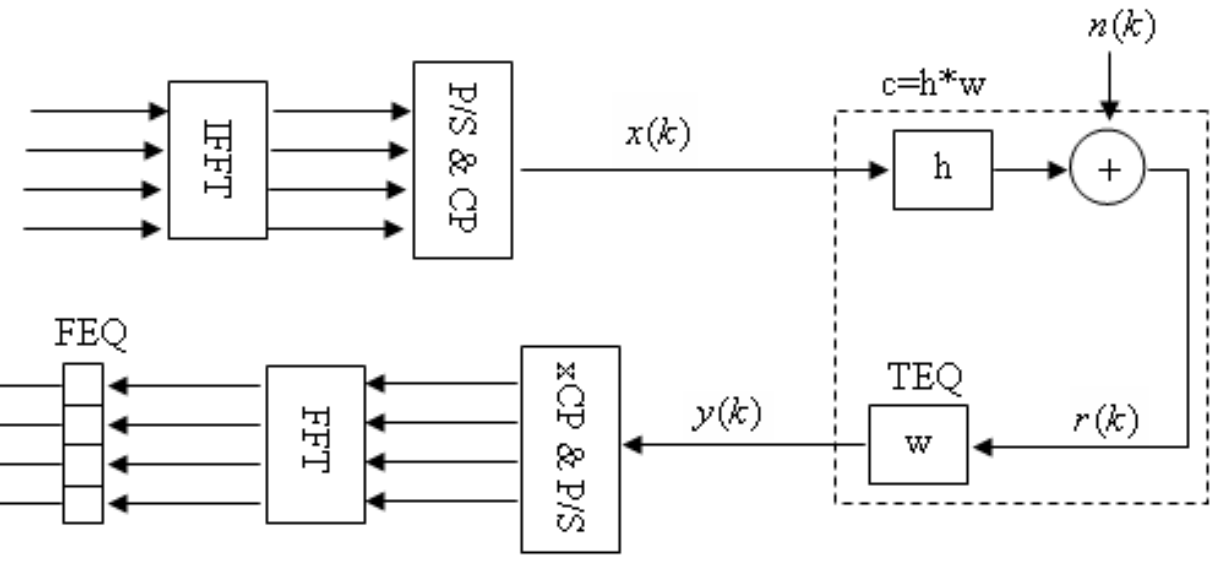

Figure 1. DFT system model. IFFT: modulation, parallel to serial (P/S), add cyclic prefix (CP), eliminate cyclic prefix $(\mathrm{xCP})$ and FFT demodulation.

For the conventional DFT-DMT system, the channel input sequence $\left[x_{n, f f t}\right]$ at the output of the IFFT block can be presented as [16]

$x_{n, f f t}=\frac{1}{N} \cdot \sum_{b=0}^{N-1} X_{b} \cdot e^{\frac{j 2 \pi b n}{N}}, \quad n=0 \quad, \quad 1 \quad, \quad \ldots \quad, \quad N-1$

where $X_{b}$ is the encoded bit stream.

When adding the $\mathrm{CP}$, the end $v$ samples are identical to the first $v$ samples in the symbol, i.e.

$x(M k+i)=x(M k+i+N), \quad i \in\{1, \cdots, v\}$

The symbol period is $M=N+v$ and $k$ is the symbol index. Figure 2 shows an example of this, with $N=8, v=2$, and $M=N+v=10$, and the symbol pictured is for $k=0$. The received data $r$ is obtained from $x$ by

$r(M k+i)=\sum_{l=0}^{L_{h}} \mathbf{h}(l) \cdot x(M k+i-l)+n(M k+i)$ 


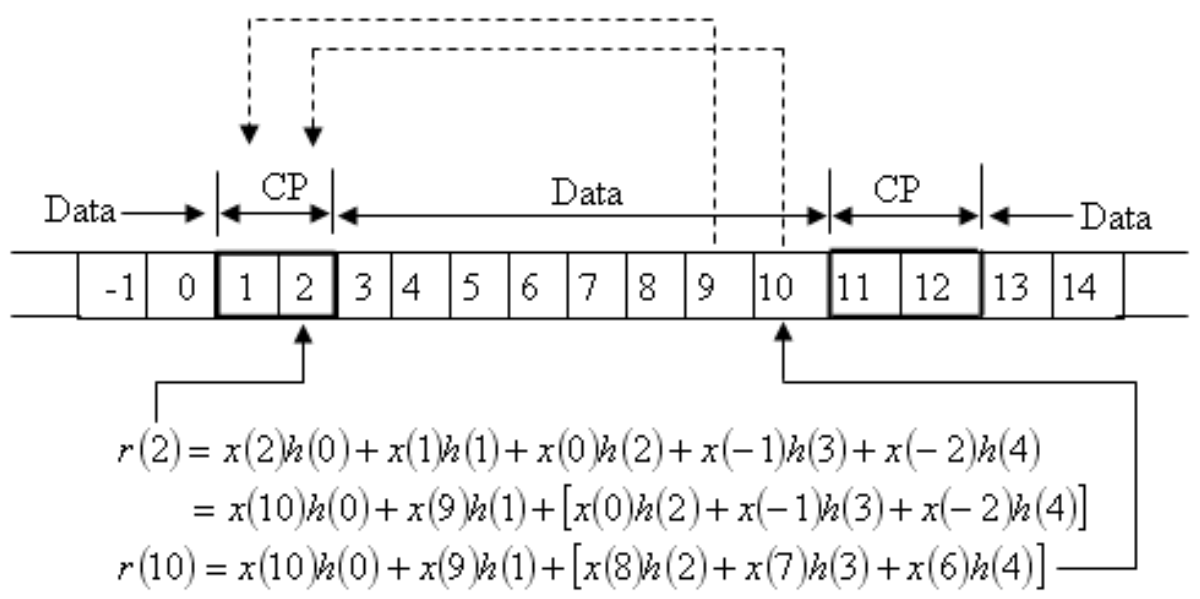

Figure 2. Demonstration of the change in the ISI at the end of the received symbol and at the received $\mathrm{CP}$.

and the equalized data $y$ is obtained from $r$ by

$$
y(M k+i)=\sum_{j=0}^{L_{w}} \mathbf{w}(j) \cdot r(M k+i-j)
$$

The channel has $L_{h}+1$ taps, the TEQ has $L_{w}+1$ taps and the effective channel $\mathbf{c}=\mathbf{h} * \mathbf{w}$ has $L_{c}+1$ taps, where $L_{c}=L_{h}+L_{w}$.

\section{Description algorithm of MERRY}

Consider the example in Figure 2. The broadcasted samples 2 and 10 are the same. But, at the receiver, the interfering samples earlier than sample 2 are not the whole identical to their correspondences before sample 10. If $h(2), h(3)$ and $h(4)$ were zero, then $r(2)=r(10)$. If we try to force $r(2)=r(10)$, we may force $h(2)=h(3)=h(4)=0$, forcing the channel selected the same like the CP. The location of the window of $v$ non-zero taps can be varied by comparing $r(3)$ to $r(11)$, or $r(4)$ to $r(12)$, etc. In general, if the channel length $L_{h}+1 \leq v$, in that case the previous sample inside the CP must look like the previous sample inside the symbol. Unit cost function that reproduce:

$$
\left.J_{\Delta}=E \llbracket y(M k+v+\Delta)-\left.y(M k+v+N+\Delta)\right|^{2}\right\}, \quad \Delta \in\{0, \cdots, M-1\}
$$

where $\Delta$ is the sign synchronization parameter, which correspond to the preferred delay of the channel-TEQ collection. The choice of $\Delta$ affects the cost function.

A randomization gradient descent of Eq.(5) guides to a sightless, adaptive TEQ, because the broadcasted data not require be identified. The proposed MERRY algorithm performs a randomization gradient descent of Eq.(5), with a limitation to keep away from the slight solution $\mathrm{w}=0$. The MERRY algorithm is summarized as follows:

For symbol $k=0,1,2, \cdots$, 
International Journal of Network Security \& Its Applications (IJNSA), Vol.5, No.6, November 2013

$$
\begin{aligned}
& \tilde{r}(k)=r(M k+v+\Delta)-r(M k+v+N+\Delta) \\
& e(k)=w^{T}(k) \tilde{r}(k) \\
& \tilde{w}(k+1)=w(k)-\mu e(k) \tilde{r}^{*}(k) \\
& w(k+1)=\frac{\tilde{w}(k+1)}{\|\tilde{w}(k+1)\|}
\end{aligned}
$$

where $r(i)=\left[r(i), r(i-1), \cdots, r\left(i-L_{w}\right)\right]^{T}$, and $*$ denotes complex conjugation.

Due to the fact that MERRY compares with CP at the last part of the symbol, one update is achievable for each symbol. Interchanging applications of the limitation have set up one tap to unity, retaining a channel approximation and renormalizing to enforce $\|c\|=1$ instead of $\|\mathrm{w}\|=1$, or with a retribution term in the cost function to put into effect the norm limitation.

\section{DST Implementation}

Applying the above algorithm to the DST approach solves the complexity problem and achieves higher bit rates than the DFT system.

For the proposed DST-DMT system, the channel input sequence $\left\{x_{n, D S T}\right\}$ at the output of the IDST block can be presented as [17]

$$
x_{n, D S T}=\left(\frac{2}{N}\right)^{\frac{1}{2}} \cdot \sum_{b=0}^{N-1} A_{b} \cdot X_{b} \cdot \sin \left[\frac{\pi b}{2 N}(2 n+1)\right] \quad, \quad n=0, \quad 1, \quad \cdots, \quad N-1
$$

where

$$
A_{b}= \begin{cases}\frac{1}{\sqrt{2}} & \text { for } \quad b=0 \\ 1 & \text { otherwise }\end{cases}
$$

, and $X_{b}$ is the encoded bit stream.

$$
\begin{aligned}
& r_{D S T}(M k+i)=\sum_{l=0}^{L_{h}} \mathbf{h}(l) \cdot x_{D S T}(M k+i-l)+n(M k+i) \\
& y_{D S T}(M k+i)=\sum_{j=0}^{L_{w}} \mathbf{w}(j) \cdot r_{D S T}(M k+i-j)
\end{aligned}
$$




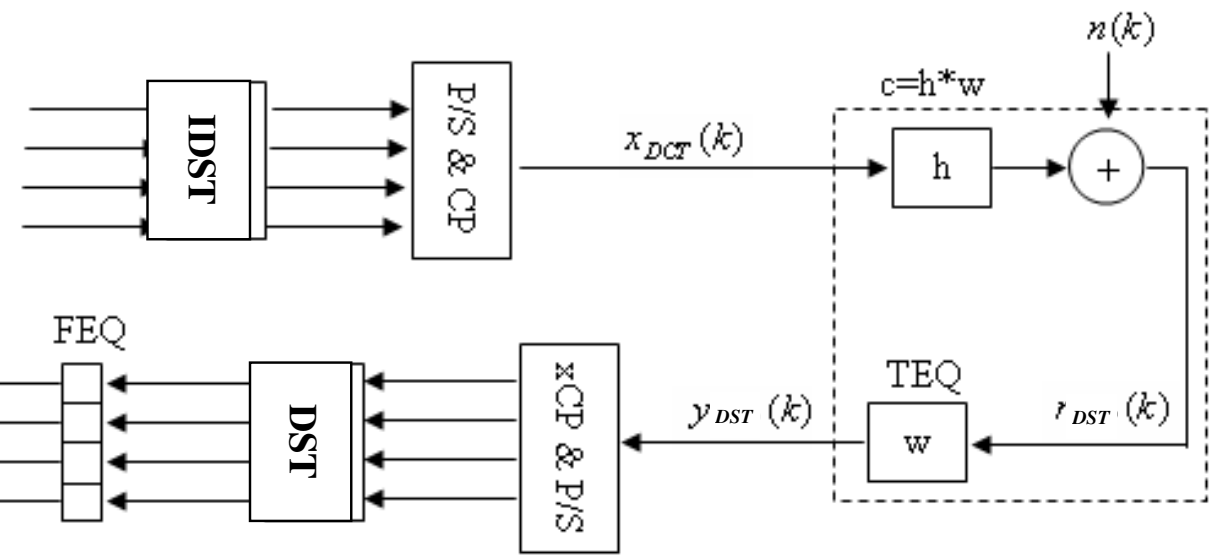

Figure 3 DST system model.

It is known that the DST has excellent spectral compaction and energy concentration properties (See Fig.(3)). It uses only real arithmetics as opposed to the complex-valued DFT. This reduces the signal processing complexity especially for real pulse-amplitude modulation signaling, where DFT-based processing still uses complex arithmetics and suffers from in-phase/quadrature imbalance problems which can cause appreciable performance degradation [11-18].

The cost function in Eq. (5) can be rewritten as:

$$
J_{\Delta, D S T}=E\left\lfloor y_{D S T}(M k+v+\Delta)-\left.y_{D S T}(M k+v+N+\Delta)\right|^{2}\right\rfloor, \quad \Delta \in\{0, \cdots, M-1\}
$$

The MERRY algorithm can be rewritten as:

For symbol $k=0,1,2, \cdots$,

$$
\begin{aligned}
& \tilde{r}_{D S T}(k)=r_{D S T}(M k+v+\Delta)-r_{D S T}(M k+v+N+\Delta) \\
& e_{D S T}(k)=w_{D S T}^{T}(k) \tilde{r}_{D S T}(k) \\
& \tilde{w}_{D S T}(k+1)=w_{D S T}(k)-\mu e_{D S T}(k) \tilde{r}_{D S T}^{*}(k) \\
& w_{D S T}(k+1)=\frac{\tilde{w}_{D S T}(k+1)}{\left\|\tilde{w}_{D S T}(k+1)\right\|}
\end{aligned}
$$

where $r_{D S T}(i)=\left[r_{D S T}(i), r_{D S T}(i-1), \cdots, r_{D S T}\left(i-L_{w}\right)\right]^{T}$, and $*$ denotes complex conjugation.

\section{Simulation parameters}

We use the standard carrier-serving-area (CSA) loop1 [15] as our test channel. The channel impulse response consists of 512 samples sampled at a rate of $2.208 \mathrm{MHz}$. Also employ high pass filter at cutoff frequency of $5.4 \mathrm{kHz}$ and pass band ripple of $0.5 \mathrm{~dB}$ to the standard loop1 to add the outcome of the splitter by the side of the transmitter. The DC channel (channel 0), channels 15 , and the Nyquist channel are not used. The DSL presentation metric is the possible bit rate for a fixed probability of error, 
International Journal of Network Security \& Its Applications (IJNSA), Vol.5, No.6, November 2013

$$
B=\sum_{i} \ln \left(1+\frac{S N R_{i}}{\Gamma}\right)
$$

When $S N R_{i}=40 \mathrm{~dB}$ in frequency bin $i$, and $\Gamma$ is the SNR gap $(\Gamma=9.8+6-4.2=11.6 \mathrm{~dB})$. This corresponds to a system margin of $6 \mathrm{~dB}$ and a coding gain of $4.2 \mathrm{~dB}$. Both the FFT size and the DST size are set to $N=512$. We assume that the power allocation is constant over all used sub-channels and that it is not changed after the TEQ is placed into the system.

\section{Simulation Results}

\section{A- for DFT Approach}

Figure 4 shows simulation results using CSA loop1. The CP size is 32. The FFT length is 512 . We have 16 taps for the TEQ. Figure 5 demonstrates a plan for bit rate opposed versus SNR. The Bit rate for this plot was computed by running for 5000 symbols and gradually decreasing the step size over time. For all these SNR values, MERRY approaches the max SSNR solution.
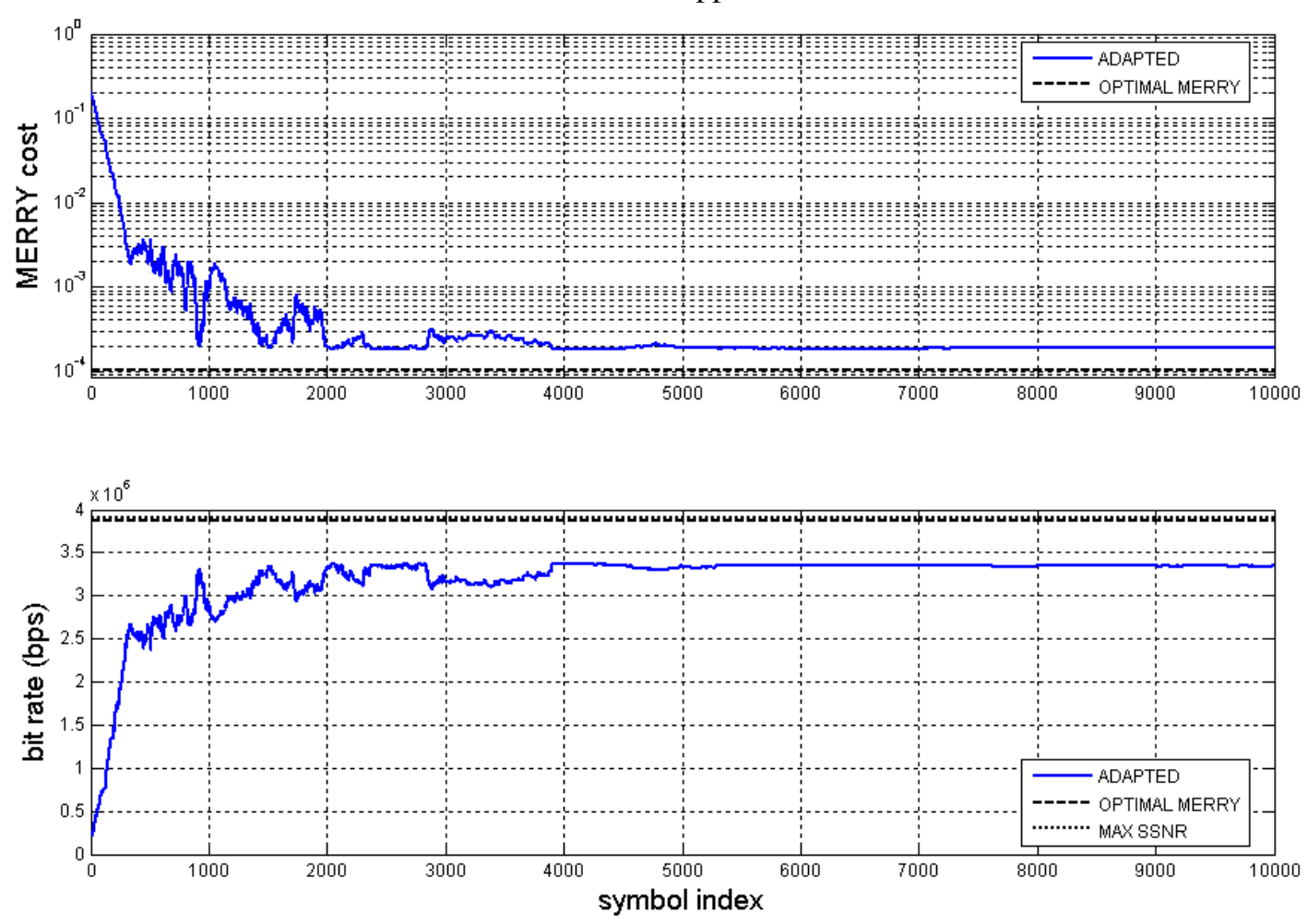

Figure 4. Achievable bit rate vs. time for the DFT system. 


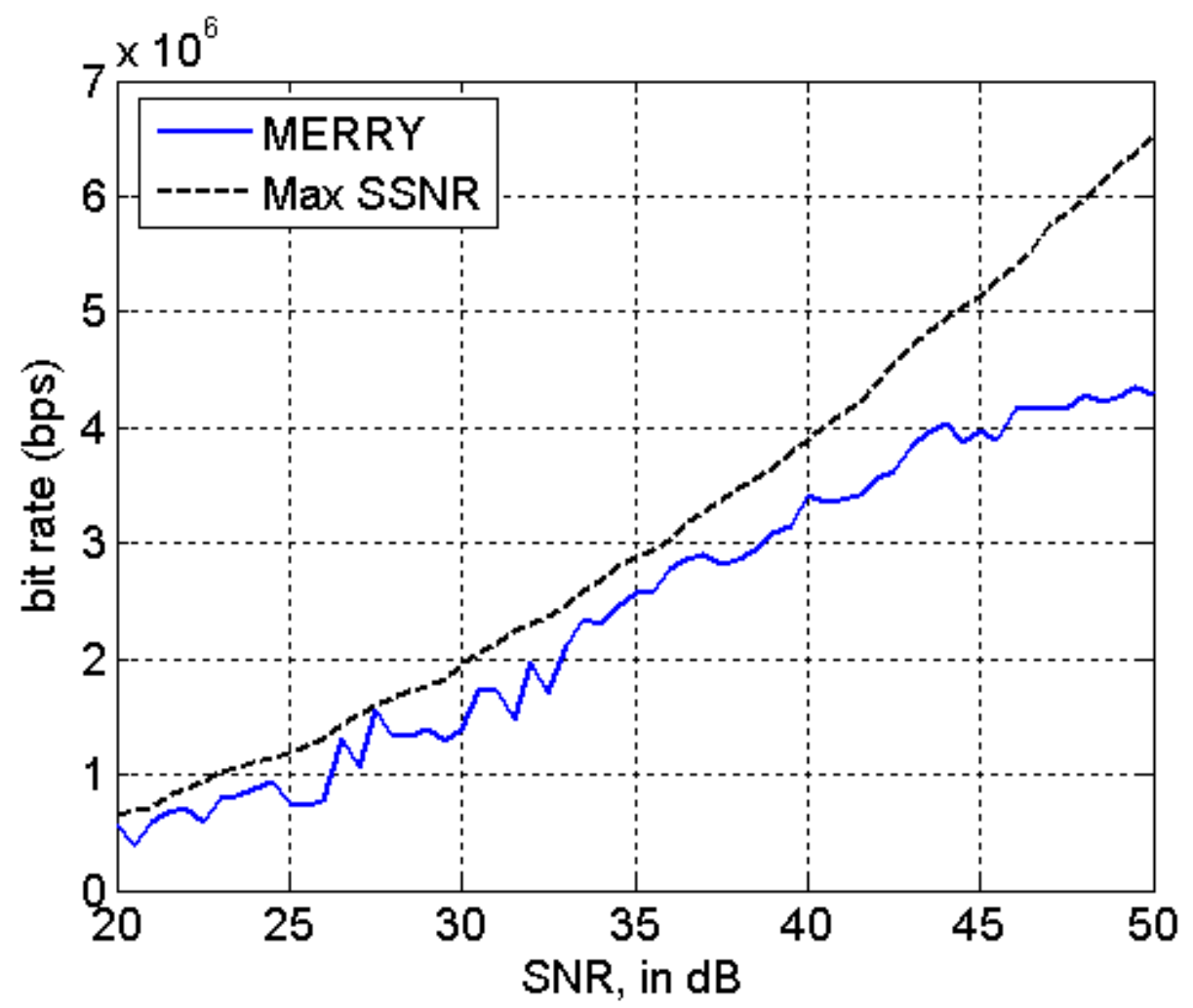

Figure 5 Achievable bit rate vs. SNR for the DFT system.

\section{B- DST System Results}

As compared to figure 4 for the DFT approach, figure 6 shows the achievable bit rate vs. time for the DST approach at the same simulation parameters. As we can see in the case of DST approach, the performance is better (high bit rate and low complexity) than that of the DFT approach.

Also, when comparing figure 5 for the DFT approach with figure 7 for the DST approach, we can see that the DST approach provides a higher bit rate than the DFT approach at the same SNR values. 
International Journal of Network Security \& Its Applications (IJNSA), Vol.5, No.6, November 2013
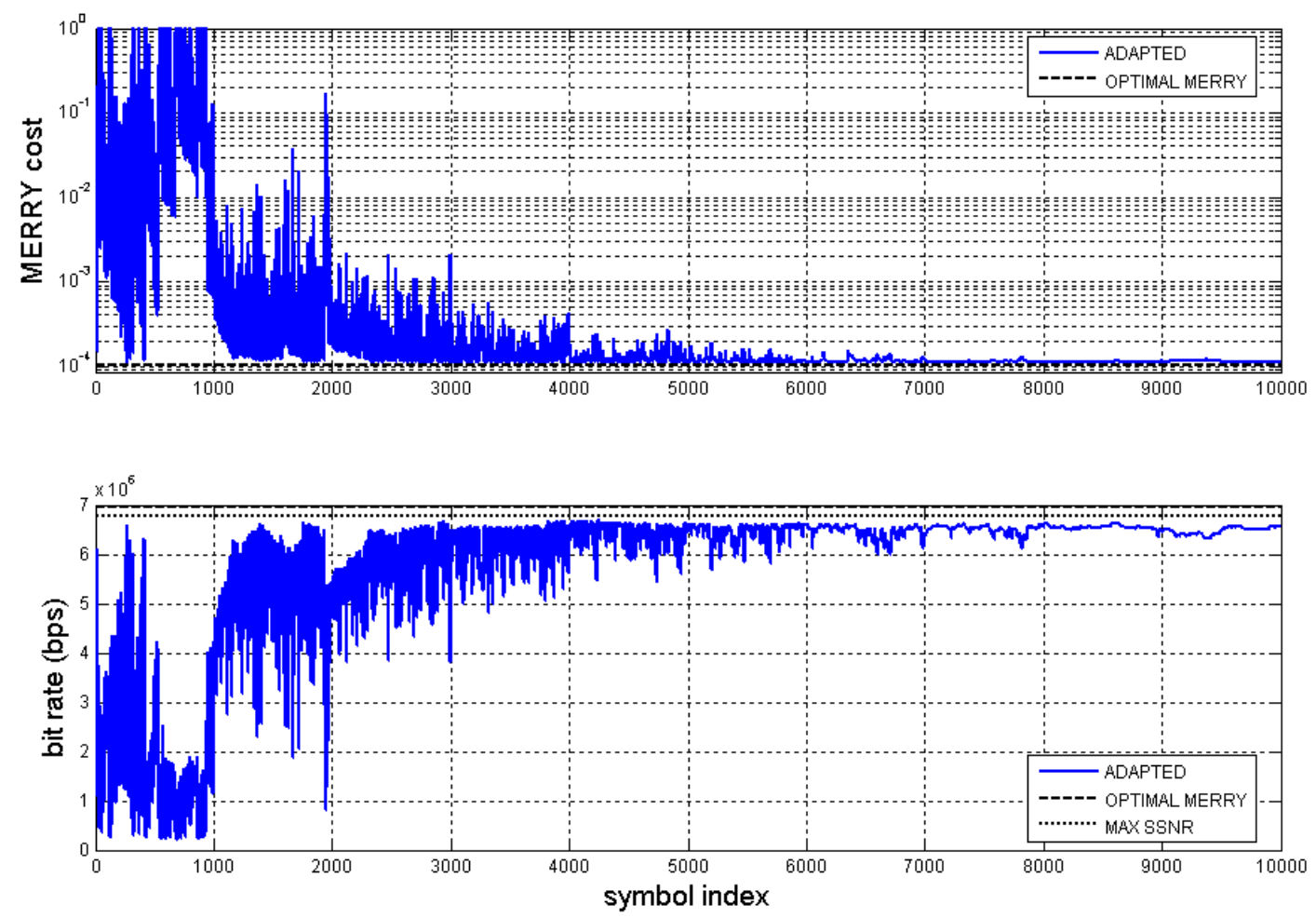

Figure 6 Achievable bit rate vs. time for the DST system.

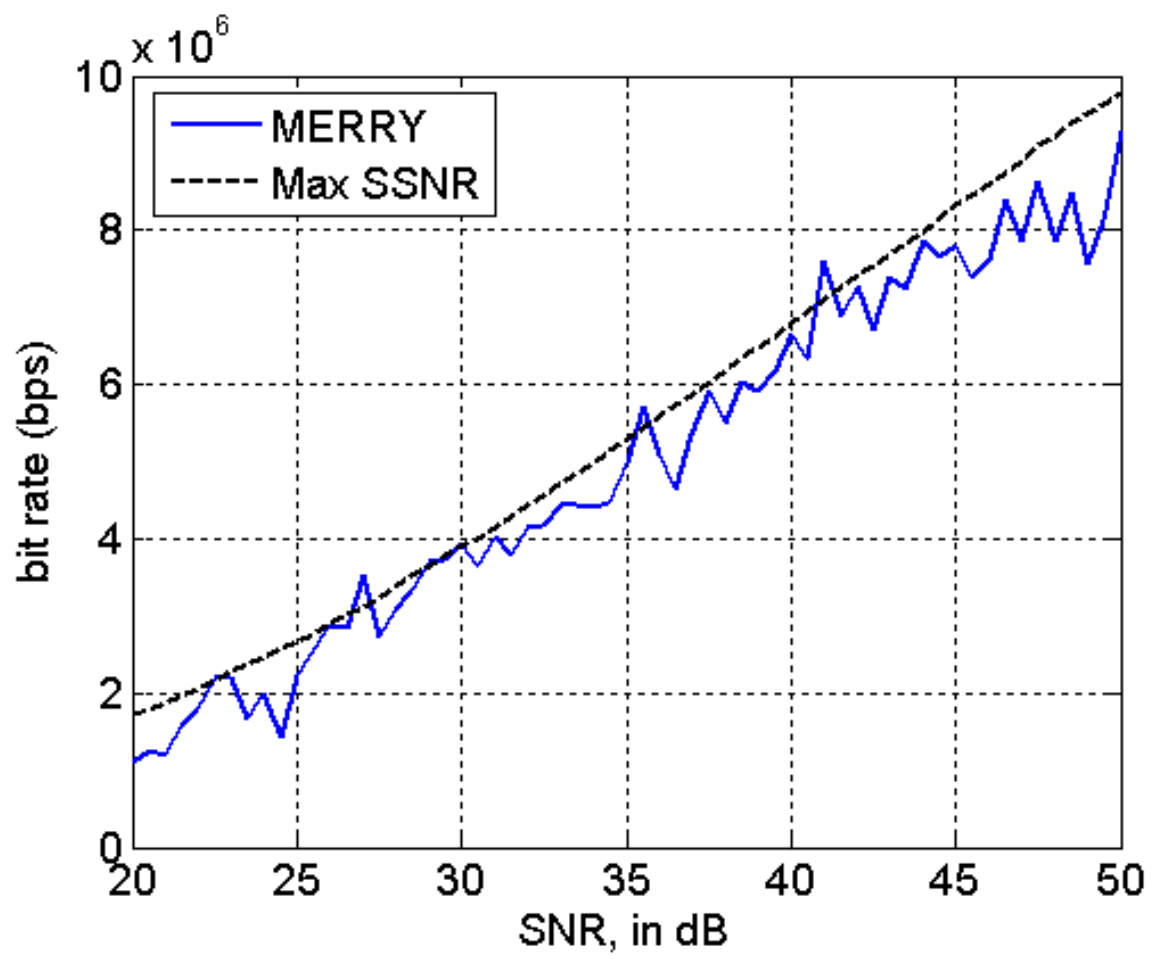

Figure 7 Achievable bit rate vs. SNR for the DST system. 
International Journal of Network Security \& Its Applications (IJNSA), Vol.5, No.6, November 2013

\section{Conclusions}

The proposed IDST/DST has been used for modulation /demodulation stages in DMT systems. Our simulation results show that the DST-DMT system provides a better performance than the DFT-DMT system because of the excellent spectral compaction and energy concentration properties of the DST compared to the DFT. It was concluded that the proposed DST-DMT system achieves higher bit rates as compared with the conventional DFT-DMT system.

\section{References}

[1] I. Lee, J. S. Chow, and J. M. Cioffi, "Performance evaluation of a fast computation algorithm for the DMT in high-speed subscriber loop," IEEE J. on Selected Areas in Comm., vol. 13, pp. 1564-1570, Dec. 1995.

[2] K. Vanble, M. Moonen, and G. Leus, "Linear and Decision-Feedback Per Tone Equalization for DMT-Based Transmission Over IIR Channels,” I IEEE Transactions Signal Processing, vol. 54, no. 1, Jan. 2006.

[3] G. Arslan, B. Lu, L. D. Clark, and B. L. Evans, "Iterative Refinement Methods for Time-Domain Equalizer Design," EURASIP Journal on Applied Signal Processing vol. 2006, pp.1-12, Aug. 2005.

[4] G. Arslan, B. L. Evans, and S. Kiaei, "Equalization for Discrete Multitone Transceivers to Maximize Bit Rate," IEEE Transactions Signal Processing, vol. 49, no. 12, pp. 3123-3135, 2001.

[5] J. S. Chow and J. M. Cioffi, "A cost-effective maximum likelihood receiver for June multicarrier systems," in Proc. IEEE Int. Conf. Comm., vol. 2, (Chicago, IL), pp. 948- 952,1992.

[6] J. S. Chow, J. M. Cioffi, and J. A. Bingham, "Equalizer training algorithms for multicarrier modulation systems," in Proc. IEEE Int. Conf. Comm., vol. 2, (Geneva, Switzerland), pp. 761-765, May 1993.

[7] N. Al-Dhahir and J. M. Cioffi, "Efficiently computed reduced-parameter input-aided MMSE equalizers for ML detection: A unified approach," IEEE Trans. on Info. Theory,vol. 42, pp. 903-915, May 1996

[8] K. Van Acker, G. Leus, M. Moonen, O. van de Wiel, and T. Pollet, "Per Tone no.1, pp. Equalization for DMT-based Systems," IEEE Transactions on Communications, vol. 49,109-119, 2001.

[9] M. Ding, Z. Shen, and B. L. Evans, "An Achievable Performance Upper Bound for Discrete Multi Tone Equalization," in Proceedings of IEEE Global Telecommunications Conference (GLOBECOM '04), vol. 4, pp. 2297-2301, Dallas, Tex, USA, November-December 2004.

[10] R. K. Martin, K. Vanbleu, M. Ding, et al., "Unification and Evaluation of Equalization Structures and Design Algorithms for Discrete Multi-tone Modulation Systems," IEEE Transactions Signal Processing, vol. 53, no. 10, part 1, pp. 3880-3894, 2005. [11] A. Rushdi and J. Tuqan, "PAPR Reduction in Trigonometric-Based OFDM Systems," IEEE Signals, Systems and Computers, Conference Record of the Forty-First Asiloma Conference pp.1747-1751, Nov. 2007.

[12] P. Tan, and N. C. Beaulieu, "A Comparison of DCT-Based OFDM and DFT-Based OFDM in Frequency Offset and Fading Channels," IEEE Transactions on Communications, vol. 54, no. 11, pp. 2113-2125, Nov. 2006.

[13] P. Tan, and N. C. Beaulieu, "An Improved DCT-Based OFDM Data Transmission Scheme," Personal, Indoor and Mobile Radio Communications, IEEE 16th International Symposium, pp.745-749, Sept. 2005.

[14] E. Khan, and C. Heneghan, "Iterative Refinement Methods for Time-Domain Equalizer Design," International Journal of Signal Processing, pp.126-130, 2006.

[15] R. K. Martin, J. Balakrishnan, W. A. Sethares, and C. R. Johnson, Jr., "A Blind, Adaptive TEQ for Multicarrier Systems," IEEE Signal Processing Letters, vol. 9, no. 11, pp. 341\{343, Nov. 2002.

[16] A.-Y. Wu T.-S. Chan, "Cost-efficient Parallel Lattice VLSI Architecture for the IFFT/FFT in DMT Transceiver Technology," IEEE, pp. 3517-3520, 1998.

[17] A. B. Watson, "Image Compression Using the Discrete Cosine Transform," Mathematica Journal, 4(1), 1994, p. 81-88.

[18] P. Tan, N. C. Beaulieu, "A Comparison of DCT-Based OFDM and DFT-Based OFDM in frequency offset and fading channels," IEEE Trans. On Commun., vol. 54, no. 11, Nov.2006. 